\title{
Nab-paclitaxel as second-line treatment in advanced gastric cancer: a multicenter phase II study of the Hellenic Oncology Research Group
}

\author{
Panagiotis Katsaounisa, Athanasios Kotsakis ${ }^{b}$, Nikolaos Kentepozidisc, Aris Polyzos ${ }^{d}$, Marios \\ Bakogeorgos ${ }^{c}$, Filippos Koinis ${ }^{b}$, Lambros Vamvakas ${ }^{b}$, Nikolaos Vardakis ${ }^{b}$, Kostas Kalbakis ${ }^{b}$, loannis \\ Boukovinas $^{\mathrm{e}}$, loannis I. Varthalitis ${ }^{f}$, Efthimios Prinarakis ${ }^{\mathrm{g}}$, Vassilis Georgoulias ${ }^{\mathrm{a}}$, John Souglakos ${ }^{\mathrm{b}}$ \\ IASO General Hospital, Athens; University General Hospital of Heraklion, Crete; 251 Air Force General Hospital, \\ Athens; Medical School, University of Athens, Laikon General Hospital, Athens; Bioclinic of Thessaloniki; Henry \\ Dunant Hospital, Athens; Hellenic Oncology Research Group, Athens, Greece
}

\section{Abstract}

Background This study evaluated the safety and efficacy of nab-paclitaxel as second-line treatment in patients with advanced gastric adenocarcinoma.

Methods Thirty-nine pretreated patients [33 with taxane-based regimens (docetaxel, cisplatin, and fluorouracil) and 6 with combination of fluoropyrimidines plus cisplatin with locally advanced inoperable and metastatic gastric and gastroesophageal junction adenocarcinoma were treated with weekly nab-paclitaxel ( $150 \mathrm{mg} / \mathrm{m}^{2} \mathrm{~d} 1, \mathrm{~d} 8$, d15 in cycles of 28 days).

Results Partial response (PR) was documented in nine patients (23.1\%; 95\% confidence interval $10.1-37.2 \%)$, stable disease (SD) in 11 (28.2\%) and disease progression in 18 (46.2\%). The disease control rate (SD + PR + complete response) was $51.3 \%$. Grade 3 and 4 neutropenia occurred in $10.2 \%$ and $5.1 \%$ of patients, respectively; grade 3 anemia in $5.1 \%$; grade 3 neurotoxicity in $5.1 \%$; and grade 2 pain in $5.1 \%$. The median progression-free survival was 3.0 months (range $0.3-13.6$ ) and the median overall survival 6.8 months (range 0.3-22).

Conclusion Nab-paclitaxel as second-line treatment in locally advanced inoperable or metastatic gastric and gastroesophageal junction carcinoma is an active chemotherapy regimen with a manageable toxicity profile and merits further evaluation.

Keywords Nab-paclitaxel, gastric cancer, second-line treatment, chemotherapy regimen

Ann Gastroenterol 2018; 31 (1): 1-7

\begin{abstract}
${ }^{a}$ Departments of Medical Oncology, IASO General Hospital, Athens (Papangiotis Katsaounis, Vassilis Georgoulias), ${ }^{b}$ Departments of Medical Oncology, University General Hospital of Heraklion, Crete (Athanasios Kotsakis, Filippos Koinis, Lambros Vamvbakas, Nikolaos Vardakis, Kostas Kalbakis, John Souglakos), 'Departments of Medical Oncology, 251 Air Force General Hospital, Athens (Nikolaos Kentepozidis, Marios Bakogeorgos), ${ }^{\mathrm{d}}$ Department of Medicine, Medical School, University of Athens, Laikon General Hospital, Athens (Aris Polyzos), ${ }^{\mathrm{e} D e p a r t m e n t s ~ o f ~}$ Medical Oncology, Bioclinic of Thessaloniki (Ioannis Boukovinas), ${ }^{\mathrm{f} D e p a r t m e n t ~ o f ~ M e d i c a l ~ O n c o l o g y, ~ H e n r y ~ D u n a n t ~ H o s p i t a l, ~ A t h e n s ~(I o a n n i s ~ I . ~}$ Varthalitis), ${ }^{g}$ Hellenic Oncology Research Group (HORG), Athens (Efthimios Prinarakis), Greece
\end{abstract}

Conflict of Interest: None

Correspondence to: John Souglakos MD, PhD, Department of Medical Oncology, University General Hospital of Heraklion, Voutes, PO Box 1352, 71110 Heraklion, Crete, Greece, e-mail: georgsec@med.uoc.gr

This work was supported by a research grant from the CELGENE International Sàrl: protocol number AX-ST-PI-0057 and the Hellenic Oncology Research Group (HORG): protocol number CT/12.05.

Number of National Ethics Committee Approval: 268/8-6-2012

Received 22 April 2017; accepted 9 October 2017;

published online 27 November 2017

DOI: https://doi.org/10.20524/aog.2017.0215 


\section{Introduction}

Gastric and gastroesophageal junction (GEJ) adenocarcinomas constitute a major health problem worldwide, representing the third most frequent cause of cancer deaths [1]. Metastatic gastric cancer is a lethal disease, with a mean overall survival (OS) of 6-12 months and 5-year survival estimate $<10 \%$ [2]. Combination chemotherapy regimens provide higher response rates than do single agents, but this translates into only modestly longer durations of disease control and survival, measured as a gain of a few weeks or months. Regimens containing docetaxel, either as monotherapy or in combination, have shown clinical activity [3]. The combination of docetaxel with cisplatin and 5-fluorouracil (5-FU), i.e. DCF regimen, results in a statistically significant improvement in response rate and survival compared to the non-docetaxel regimen (cisplatin and 5-FU alone); however, the DCF regimen is associated with increased toxicity $[4,5]$. In addition, the replacement of cisplatin with oxaliplatin in the r5-FU, leucovorin, oxaliplatin, and docetaxel (FLOT) regimen as weekly administration has shown comparable efficacy with more favorable toxicity results [6]. Given the encouraging results of DCF and FLOT, these regimens are usually administered nowadays in several countries as first-line treatment. On the other hand, the second-line treatment options are limited. In general, clinical trials assessing the efficacy of a variety of secondline therapy regimens have shown lower response rates and a poorer toxicity profile compared to first-line regimens [7-11]. Weekly paclitaxel [6], irinotecan as a single agent [11] or in combination with 5-FU [10] were the preferable regimens in the most part of the world. Ramucirumab is monoclonal antibody that binds to vascular endothelial growth factor receptor (VEGFR)-2, blocking receptor activation. Recently, the addition of ramucirumab to weekly paclitaxel has shown an increase in response rate, progression-free survival (PFS) and OS in a randomized prospective study [12].

Nab-paclitaxel is a novel nanoparticle form of paclitaxel bound with human albumin. Clinical studies with nabpaclitaxel, in patients with metastatic breast cancer, demonstrated that the lack of solvents during the preparation of nab-paclitaxel offers many advantages during intravenous administration such as: i) a higher maximum tolerated dose $\left(300 \mathrm{mg} / \mathrm{m}^{2}\right.$ vs $\left.175 \mathrm{mg} / \mathrm{m}^{2}\right)$; ii) no premedication with corticosteroids; iii) no need for special filters, used for solventbased paclitaxel; and iv) an increased infusion rate.

In a phase III study of patients with metastatic breast cancer, nab-paclitaxel demonstrated superior efficacy, with higher response rates, a longer time to progression (TTP), and higher OS in comparison to solvent-based paclitaxel [13]. Based on these favorable pharmacologic characteristics of nab-paclitaxel, the gastrointestinal working group of the Hellenic Oncology Research Group (HORG) designed a phase II study in which nabpaclitaxel was administered as salvage second-line treatment in patients with advanced gastric and GEJ adenocarcinomas.

\section{Patients and methods}

\section{Patient eligibility}

Patients with histologically or cytologically confirmed inoperable locally advanced, recurrent or metastatic gastric or GEJ adenocarcinomas, with documented disease progression after first-line systemic treatment, were enrolled in the study. Additional inclusion criteria included bidimensional measurable disease, age $>18$ years, ECOG performance status (PS) of 0-2, adequate bone marrow, liver and renal function, a life expectancy of at least 3 months and written informed consent. Exclusion criteria included a locally advanced carcinoma of the stomach and GEJ not corresponding to local disease, active gastrointestinal hemorrhage, obstruction, known brain metastases, active infection, clinically significant cardiovascular disease, pregnancy and lactation, as well as psychiatric disorders.

The study was approved by the National Drug Administration, the National Ethics Committee, as well as the Ethics Committees of the participating institutions.

\section{Patient evaluation}

Pre-treatment evaluation included a complete medical history, physical examination and blood pressure measurement; a complete blood count (CBC) with differential and platelet count; standard biochemical profile; electrocardiogram (ECG); chest X-rays; and computed tomography scans of the chest and abdomen. Brain and bone scintigraphy were performed only in patients with suspicious symptoms. During treatment, a CBC was performed weekly, while in case of grade 3 and 4 neutropenia and thrombocytopenia the $\mathrm{CBC}$ was recorded daily until hematological recovery. A detailed medical history was taken and a complete physical examination was performed before each course of treatment to document symptoms of disease and chemotherapy-related toxicities. Biochemical tests, CBC and ECG were performed every 3 weeks. Lesions assessable by computed tomography scans were evaluated after every 2 courses of treatment using the Response Evaluation Criteria in Solid Tumors (RECIST)1.1 [14].

\section{Treatment}

Nab-paclitaxel (Abraxane ${ }^{\circledR}$, Celgene) was given at a dose of $150 \mathrm{mg} / \mathrm{m}^{2}$ on days 1,8 and 15 of each chemotherapy cycle (28-day cycles) until disease progression. This dose was derived from previous studies of metastatic breast cancer $[15,16]$. Treatment was administered until disease progression and doseadjustment criteria were based on hematological parameters. The dosage of nab-paclitaxel was reduced by $25 \mathrm{mg} / \mathrm{m}^{2}$ in the subsequent cycle in case of febrile neutropenia or grade 4 neutropenia or thrombocytopenia lasting for more than 5 days. A one-week treatment delay and/or a $25 \mathrm{mg} / \mathrm{m}^{2}$ dose reduction of nab-paclitaxel was performed in patients with more than 
grade 2 non-hematological toxicity, with the exception of alopecia and nausea. Dose reductions were maintained for all subsequent treatment cycles and up to 2 dose level reductions were allowed. Treatment delays up to 14 days or the need for more than 2 dose level reductions were grounds for drug discontinuation. Toxicity was graded according to the NCICommon Terminology Criteria for adverse events (NCTCAE: version 4.0). Prophylactic administration of recombinant human granulocyte colony-stimulating factor was not allowed.

\section{Statistical analysis}

The study was an open-label, single-arm, multicenter phase II trial, conducted in 11 Greek centers. The primary endpoint of the study was the overall response rate (ORR) and the secondary endpoints were PFS, OS, and the toxicity profile. The study followed Fleming's two-stage design for clinical phase II studies. The sample size calculation was based on the assumption that the expected ORR would be $20 \%$ (based on the data from the literature for second-line treatment in gastric and GEJ adenocarcinomas $[6,10]$ ) and the minimum acceptable response 7\%; if at least two responses were observed among the first 26 enrolled patients during the first part of the study, then 13 additional patients had to be enrolled in the second part of the study, for a total of 39 patients [17]. The null hypothesis would be rejected, with $5 \%$ probability and $80 \%$ statistical power, if at least 5 responses were observed in 39 enrolled patients.

All patients who received at least one cycle of treatment were evaluable for analysis. OS and PFS were calculated from the date of randomization until the date of death and the date of documentation of disease progression, respectively. Qualitative variables were compared using the chi-square test. A P-value $<0.05$ was considered to indicate statistical significance. Continuous variables are summarized in frequency tables.

\section{Results}

\section{Patient characteristics}

Between January 2012 and April 2015, a total of 39 patients were enrolled in the study. Baseline patient characteristics are listed in Table 1. In brief, patients' median age was 62 years (range 24-79), 29 (74.4\%) were men and all had an ECOG PS of $0-1$; histology was diffuse adenocarcinoma in 12 patients (30.8\%), intestinal type adenocarcinoma in 16 patients $(41 \%)$ and indeterminate type in 11 patients $(28.2 \%)$. Four patients (10.2\%) had received adjuvant treatment and 17 (43.6\%) had initially undergone surgical resection. Thirty-three (84.6\%) patients had received first-line chemotherapy with taxane-based regimens (DCF) while 6 patients were under treatment with a combination of fluoropyrimidines plus cisplatin. Nine (23.1\%) patients had primary resistant disease (relapse $<3$ months) to first-line chemotherapy, while 30 (76.9\%) had refractory disease.
Table 1 Demographic data

\begin{tabular}{|c|c|}
\hline Characteristic & $\begin{array}{l}\text { Number of } \\
\text { patients (\%) }\end{array}$ \\
\hline \multicolumn{2}{|l|}{ Age } \\
\hline Median (range) & $62.0(24-79)$ \\
\hline \multicolumn{2}{|l|}{ Sex } \\
\hline Male & $29(74.4)$ \\
\hline Female & $10(25.6)$ \\
\hline \multicolumn{2}{|l|}{ Performance status } \\
\hline 0 & $11(28.2)$ \\
\hline 1 & $28(71.8)$ \\
\hline \multicolumn{2}{|l|}{ Grade } \\
\hline 2 & $14(35.9)$ \\
\hline 3 & $20(51.3)$ \\
\hline Undefined & $1(2.6)$ \\
\hline Unknown & $4(10.2)$ \\
\hline \multicolumn{2}{|l|}{ Histology } \\
\hline Diffuse type adenocarcinoma & $12(30.8)$ \\
\hline Intestinal type adenocarcinoma & $16(41.0)$ \\
\hline Indeterminate type adenocarcinoma & $11(28.2)$ \\
\hline \multicolumn{2}{|l|}{ Prior surgery } \\
\hline Radical gastrectomy & $13(33.4)$ \\
\hline Partial gastrectomy & $4(10.2)$ \\
\hline No & $22(56.4)$ \\
\hline \multicolumn{2}{|l|}{ Adjuvant treatment } \\
\hline Yes & $4(10.2)$ \\
\hline No & $35(89.8)$ \\
\hline \multicolumn{2}{|l|}{ Prior treatment for metastatic disease } \\
\hline Taxane-based (DCF) & $33(84.6)$ \\
\hline $\begin{array}{l}\text { Non-taxane-based (fluoropyrimidines } \\
\text { plus cisplatin) }\end{array}$ & $6(15.4)$ \\
\hline \multicolumn{2}{|l|}{ Prior response to first-line treatment } \\
\hline $\mathrm{CR} / \mathrm{PR}$ & $22(56.4)$ \\
\hline SD & $9(23.1)$ \\
\hline $\mathrm{PD}$ & $8(20.5)$ \\
\hline \multicolumn{2}{|l|}{$\begin{array}{l}\text { Type of chemoresistance to first-line } \\
\text { treatment }\end{array}$} \\
\hline Resistant tumors & $9(23.1)$ \\
\hline Refractory tumors & $30(76.9)$ \\
\hline
\end{tabular}

$\overline{C R / P R}$, complete/partial response; $S D$, stable disease; $P D$, disease progression; $D C F$, docetaxel with cisplatin and 5-fluorouracil

\section{Compliance with treatment}

A total of 123 chemotherapy cycles were administered with a median of 2 cycles per patient (range 1-8). Dose reduction was required in 28 cycles $(23.6 \%)$, because of hematological 
( $\mathrm{n}=12$ cycles) and non-hematological ( $\mathrm{n}=16$ cycles) toxicity. Dose reduction because of neurotoxicity was required in 4 cycles (3.2\%). Thirty-nine cycles (31.7\%) were delayed by a maximum of 5 days: for personal reasons unrelated to treatment or disease, in response to patients' request or for reasons related to radiological assessment ( $\mathrm{n}=16$ cycles); hematological toxicity ( $\mathrm{n}=9$ cycles); or non-hematological toxicity ( $\mathrm{n}=14$ cycles). At the time of analysis, 31 patients $(79.5 \%)$ discontinued treatment because of disease progression, three patients $(7.7 \%)$ because of treatment-related adverse events; four patients (10.3\%) refused further treatment; and one patient (2.6\%) died for reasons unrelated to the disease or treatment. The mean dose intensity for nab-paclitaxel was $100 \mathrm{mg} /$ $\mathrm{m}^{2} /$ week, corresponding to $89 \%$ of the protocol-predicted dose.

\section{Efficacy}

A total of 38 patients were evaluable for response. One patient was not evaluable for response since he died suddenly for reasons unrelated to the disease (cardiac arrest). Partial response (PR) was documented in nine patients (23.1\%; 95\% confidence interval [CI] 10.1-37.2\%); stable disease (SD) in 11 (28.6\%); and disease progression in 18 (53.9\%). Response rates were similar in patients with refractory disease and disease resistant to first-line chemotherapy ( 2 of $9,22.2 \%$, and 7 of $30,23.3 \%$, respectively). The disease control rate $(\mathrm{PR}+\mathrm{SD})$ was $51.3 \%$. There was no correlation between the response rate and the patient's PS or the

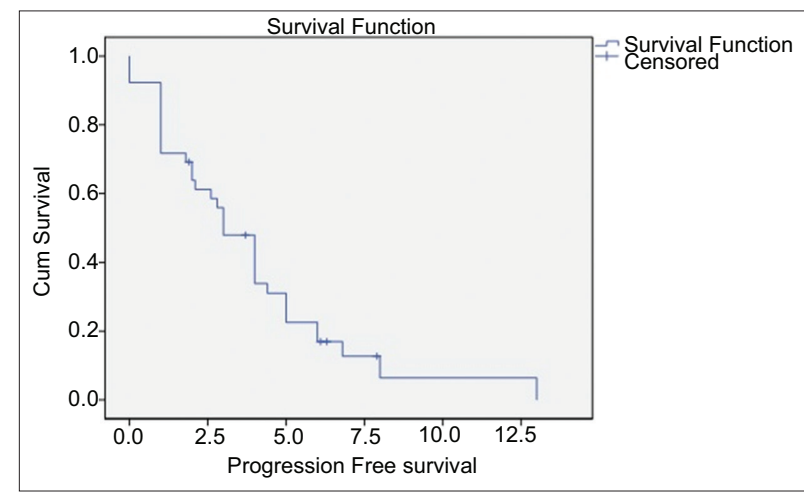

Figure 1 Kaplan-Meier curve for estimating progression-free survival

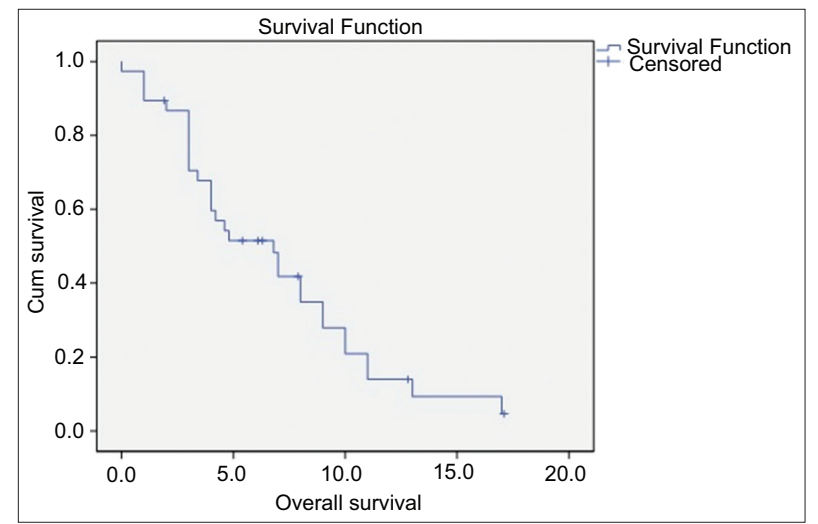

Figure 2 Kaplan-Meier curve for estimating overall survival stage of the disease. The median PFS (mPFS) was 3 months (range $0.3-13.6)$ (Fig. 1). At the time of analysis, 31 patients (79.5\%) had died as a consequence of disease progression. The median OS (mOS) was 6.8 months (range 0.3-22.0) (Fig. 2). These efficacy results (ORR, mPFS and mOS) were not different in the group of patients $(n=33)$ who had received first-line chemotherapy with docetaxel-based chemotherapy (DCF regimen).

\section{Safety}

All patients who received at least one cycle of chemotherapy were evaluable for toxicity. Most of them reported only mild adverse events (grade 1-2). The most common grade 1-2 adverse events were neutropenia (66.7\%), anemia (87.2\%), and fatigue (41\%). Grade $3-4$ neutropenia occurred in $10.2 \%$ and $5.1 \%$ of the patients, respectively and grade 3 anemia in 2 patients (5.1\%). The most common grade 3-4 non-hematological toxicities were vomiting (7.7\%), neurotoxicity (5.1\%), and pain (5.1\%) (Table 2).

\section{Discussion}

For patients with gastric and GEJ adenocarcinomas, who retain a good PS, there are limited therapeutic options for second-line therapy. Several anti-tumor drugs have been tested in this setting; however, no "clear" winner has been identified and few trials have compared different agents. The response rates are low and the OS disappointing $[17,18]$.

This study aimed to evaluate a new treatment option as second-line therapy for patients with advanced gastric and GEJ adenocarcinomas. This multicenter study met its primary endpoint, i.e. the ORR. Indeed, the administration of nabpaclitaxel resulted in an ORR of $23.1 \%$ and a disease control rate of $51.3 \%$; moreover, the mPFS reached 3.0 months, and the mOS 6.8 months. These efficacy results compare favorably with those achieved in the arm with weekly paclitaxel single agent (ORR 16\%, PFS 2.9 months, and OS 7.4 months) in the RAINBOW trial [12], or those with single-agent irinotecan (ORR 17.2\%, PFS 2.2 months, and OS 5.8 months), or the FOLFIRI regimen (ORR 20\%, PFS 3.0 months, and OS 6.7 months)[19], or singleagent ramucirumab (PFS 2.1 months, and OS 5.2 months) in the REGARD trial [20]. In contrast, the addition of ramucirumab to weekly paclitaxel achieved a significant improvement in PFS (4.4 months) and OS (9.2 months)[12]. We should underline that all but three patients had previously been treated with a taxanebased regimen DCF, whereas all patients were taxane-naïve in the RAINBOW trial [12]. In addition, the regimen had an expected and manageable toxicity profile, with already pre-described adverse events of single-agent nab-paclitaxel in other solid tumor studies $[15,16]$. Taking all the abovementioned data into perspective, we can conclude that nab-paclitaxel in combination with ramucirumab merits further evaluation in taxane-naïve and taxane-pretreated patients in randomized trials.

In the current study, nab-paclitaxel proved its efficacy, even in this taxane-based pretreated group of patients. Its efficacy 
Table 2 Adverse events related to study treatment

\begin{tabular}{|c|c|c|c|c|}
\hline Adverse Event & Grade I N (\%) & Grade II N (\%) & Grade III N (\%) & Grade IV N \\
\hline Leukopenia & $8(20.5)$ & $8(20.5)$ & $3(7.7)$ & \\
\hline Nausea & $6(15.4)$ & $3(7.7)$ & & \\
\hline Lymphopenia & $1(2.6)$ & & $1(5.2)$ & \\
\hline Vomiting & $6(15.4)$ & $2(5.2)$ & $3(7.7)$ & \\
\hline Neutropenia & $14(35.9)$ & $12(30.8)$ & $4(10.2)$ & $2(5.2)$ \\
\hline Fatigue & $10(25.6)$ & $6(15.4)$ & $1(2.6)$ & \\
\hline Pain & $8(20.5)$ & & $2(5.2)$ & \\
\hline Alopecia & $1(2.6)$ & $8(20.5)$ & & \\
\hline Neurotoxicity & $7(18.0)$ & $6(15.4)$ & $2(5.2)$ & \\
\hline Anemia & $22(56.4)$ & $12(30.8)$ & $2(5.2)$ & \\
\hline Constipation & $6(15.4)$ & $1(2.6)$ & & \\
\hline Dermatitis & $2(5.2)$ & $1(2.6)$ & & \\
\hline Diarrhea & $1(2.6)$ & $2(5.2)$ & & \\
\hline Thrombocytopenia & $1(2.6)$ & & & \\
\hline Mucositis & $1(2.6)$ & $1(2.6)$ & & \\
\hline Respiratory infection & $1(2.6)$ & & & \\
\hline Hyperglycemia & $4(10.2)$ & $1(2.6)$ & & \\
\hline
\end{tabular}

\section{Summary Box}

\section{What is already known:}

- Second-line treatment of patients with advanced/ metastatic gastric and gastro-esophageal adenocarcinomas is an unmet need

- Weekly paclitaxel in combination with ramucirumab is the preferable treatment of choice in this setting

- Single-agent paclitaxel or irinotecan as well as the FOLFIRI regimen are considered as relevant choices

\section{What the new findings are:}

- In the current study nab-paclitaxel was proven effective in taxane-pretreated patients and had an acceptable toxicity profile

- The efficacy of nab-paclitaxel in taxane-pretreated patients was comparable with those reported with single-agent paclitaxel or irinotecan, or FOLFIRI, but inferior to those obtained with the paclitaxelramucirumab combination in taxane-naïve patients

- Randomized trials are warranted for the evaluation of nab-paclitaxel plus ramucirumab in taxanepretreated and taxane-naïve patients may be explained by the ability of nab-paclitaxel to take advantage of the gp60 and caveolae-mediated albumin transport pathway to traverse the blood vessel endothelial lining into the tumor, wherein it may be referentially retained by tumoral SPARC [21,22]. Indeed, studies in nude mice bearing human tumor xenografts showed that mice could tolerate higher doses of nab-paclitaxel compared to Cremophor-paclitaxel, leading to more complete regressions and longer survival in the nabpaclitaxel-treated groups. These findings have been confirmed in clinical trials. Nowadays, apart from metastatic breast cancer, successful clinical trials have confirmed the activity of nabpaclitaxel in other tumor types, such as metastatic pancreatic adenocarcinoma and non-small-cell lung cancer of squamous origin [23-25]. Moreover, the absence of solvents, such as Cremophor, in conventional paclitaxel, makes it a much more patient-friendly taxane, since it is administered in a much shorter time without the need for premedication. Nevertheless, most of the patients relapsed early after their initial response, indicating a shared mechanism of resistance in all taxanes. The elucidation of this resistance mechanism may be the key for the rational design of treatment strategy in advanced or metastatic gastric cancer.

Today, in the era of targeted therapies, only ramucirumab, an anti-VEGFR-2 monoclonal antibody, is currently approved as second-line treatment, as monotherapy or in combination with paclitaxel in advanced gastric cancer. In the monotherapy trial, the benefits were modest, with PFS and OS 2.1 months and 5.8 months, respectively, and ORR 8\% [20]. Even in the combination trial, where the results were more encouraging (PFS and OS 4.4 and 9.6 months, respectively, ORR 28\%), we should take into account that the population treated was taxane-naïve [12]. 
Recently, an open-label, randomized, non-inferiority, multicenter Japanese trial has been reported, which compared solvent-based paclitaxel with nab-paclitaxel [26]. Patients were randomly assigned 1:1:1 to receive intravenous nabpaclitaxel on day 1 of each 3-week cycle, nab-paclitaxel on days 1,8 , and 15 of each 28 -day cycle, or solvent-based paclitaxel on days 1,8 , and 15 of each 28-day cycle after progression to fluoropyrimidine-based first-line treatment. The study reported that weekly nab-paclitaxel was non-inferior to weekly solvent-based paclitaxel (hazard ratio 0.97; 95\%CI 0.76-1.23; $\mathrm{P}=0.0085)$, with a mOS of 11.1 months $(95 \% \mathrm{CI}$ 9.9-13.0) for weekly nab-paclitaxel, and 10.9 months (95\%CI 9.4-11.8) for weekly solvent-based paclitaxel. Although, the mOS was higher than that reported in the current study, this difference could be attributed to differences in patients' ethnicity and disease characteristics, and more importantly to the fact that the vast majority of patients in our study had progressed after taxane-containing therapy in the first-line setting.

On the other hand, the results of the present study should be interpreted with caution (small sample size, possible selection bias in a phase II study) and can only serve as a hypothesisgenerating trial. Nab-paclitaxel proved its efficacy in taxaneresistant/refractory tumors, with results comparable to those of single-agent weekly paclitaxel or biweekly irinotecan, as well as the FOLFIRI regimen. However, these results are inferior to the combination of weekly paclitaxel plus ramucirumab in taxanenaive patients; thus, the evaluation of nab-paclitaxel plus ramucirumab in taxane-pretreated and taxane-naïve patients is the next logical step.

In conclusion, this phase II study demonstrated that nab-paclitaxel as second-line treatment in locally advanced inoperable or metastatic gastric and GEJ carcinoma is effective and merits further evaluation in subsequent clinical trials.

\section{Acknowledgments}

We would like to sincerely thank Dora Hatzidaki, Eva Maragoudaki, Ioannis Athanasakis, Marina Mavrogianni, Vassiliki Schoina, Aspasia Dimou, Spiros Georgiadis, Ageliki Kalisperi and Vasso Athanasaki for their contribution in this research.

\section{References}

1. Siegel RL, Miller KD, Jemal A. Cancer statistics, 2015. CA Cancer J Clin 2015;65:5-29.

2. Hundahl SA, Phillips JL, Menck HR. The National Cancer Data Base Report on poor survival of U.S. gastric carcinoma patients treated with gastrectomy: Fifth Edition American Joint Committee on Cancer staging, proximal disease, and the "different disease" hypothesis. Cancer 2000;88:921-932.

3. Mavroudis D, Kourousis C, Androulakis N, et al. Frontline treatment of advanced gastric cancer with docetaxel and granulocyte colony-stimulating factor (G-CSF): a phase II trial. Am J Clin Oncol 2000;23:341-344.
4. Ajani JA, Fodor MB, Tjulandin SA, et al. Phase II multiinstitutional randomized trial of docetaxel plus cisplatin with or without fluorouracil in patients with untreated, advanced gastric, or gastroesophageal adenocarcinoma. J Clin Oncol 2005;23:5660-5667.

5. Van Cutsem E, Moiseyenko VM, Tjulandin S, et al. Phase III study of docetaxel and cisplatin plus fluorouracil compared with cisplatin and fluorouracil as first-line therapy for advanced gastric cancer: a report of the V325 Study Group. J Clin Oncol 2006;24:4991-4997.

6. Al-Batran SE, Homann N, Pauligk C, et al. Effect of neoadjuvant chemotherapy followed by surgical resection on survival in patients with limited metastatic gastric or gastroesophageal junction cancer: The AIO-FLOT3 Trial. JAMA Oncol 2017;3:1237-1244.

7. Kodera Y, Ito S, Mochizuki Y, et al; Chubu Clinical Cancer Group. A phase II study of weekly paclitaxel as second-line chemotherapy for advanced gastric Cancer (CCOG0302 study). Anticancer Res 2007;27:2667-2671.

8. Lorenzen S, Duyster J, Lersch C, et al. Capecitabine plus docetaxel every 3 weeks in first- and second-line metastatic oesophageal cancer: final results of a phase II trial. Br J Cancer 2005;92:2129-2133.

9. Hartmann JT, Pintoffl JP, Al-Batran SE, et al. Mitomycin C plus infusional 5-fluorouracil in platinum-refractory gastric adenocarcinoma: an extended multicenter phase II study. Onkologie 2007;30:235-240.

10. Sym SJ, Ryu MH, Lee JL, et al. Salvage chemotherapy with biweekly irinotecan, plus 5-fluorouracil and leucovorin in patients with advanced gastric cancer previously treated with fluoropyrimidine, platinum, and taxane. Am J Clin Oncol 2008;31:151-156.

11. Chun JH, Kim HK, Lee JS, et al. Weekly irinotecan in patients with metastatic gastric cancer failing cisplatin-based chemotherapy. Jpn J Clin Oncol 2004;34:8-13.

12. Wilke H, Muro K, Van Cutsem E, et al; RAINBOW Study Group. Ramucirumab plus paclitaxel versus placebo plus paclitaxel in patients with previously treated advanced gastric or gastrooesophageal junction adenocarcinoma (RAINBOW): a doubleblind, randomised phase 3 trial. Lancet Oncol 2014;15:1224-1235.

13. Gradishar WJ, Tjulandin S, Davidson N, et al. Phase III trial of nanoparticle albumin-bound paclitaxel compared with polyethylated castor oil-based paclitaxel in women with breast cancer. J Clin Oncol 2005;23:7794-7803.

14. Eisenhauer EA, Therasse P, Bogaerts J, et al. New response evaluation criteria in solid tumours: revised RECIST guideline (version 1.1). Eur J Cancer 2009;45:228-247.

15. Aapro M, Tjulandin S, Bhar P, Gradishar W. Weekly nab-paclitaxel is safe and effective in $\geq 65$ years old patients with metastatic breast cancer: a post-hoc analysis. Breast 2011;20:468-474.

16. Dent S, Fraser J, Graham N, Campbell M, Hopkins S, Dranitsaris G. Clinical outcomes of women with metastatic breast cancer treated with nab-paclitaxel: experience from a single academic cancer centre. Curr Oncol 2013;20:24-29.

17. Thallinger CM, Raderer M, Hejna M. Esophageal cancer: a critical evaluation of systemic second-line therapy. J Clin Oncol 2011;29:4709-4714.

18. Wesolowski R, Lee C, Kim R. Is there a role for second-line chemotherapy in advanced gastric cancer? Lancet Oncol 2009; 10:903-912.

19. Sym SJ, Hong J, Park J, et al. A randomized phase II study of biweekly irinotecan monotherapy or a combination of irinotecan plus 5 -fluorouracil/leucovorin (mFOLFIRI) in patients with metastatic gastric adenocarcinoma refractory to or progressive after first-line chemotherapy. Cancer Chemother Pharmacol 2013;71:481-488.

20. Fuchs CS, Tomasek J, Yong CJ, et al; REGARD Trial Investigators. Ramucirumab monotherapy for previously treated advanced gastric or gastro-oesophageal junction adenocarcinoma (REGARD): an international, randomised, multicentre, placebo-controlled, phase 
3 trial. Lancet 2014;383:31-39.

21. Zeng J, Yin J, Yuan D, et al. Assessing the clinical outcome of nabpaclitaxel in Chinese patients with advanced non-small-cell lung cancer. Clin Respir J 2017;11:632-639.

22. Zhang L, Marrano P, Kumar S, et al. Nab-paclitaxel is an active drug in preclinical model of pediatric solid tumors. Clin Cancer Res 2013;19:5972-5983.

23. Von Hoff DD, Ervin T, Arena FP, et al. Increased survival in pancreatic cancer with nab-paclitaxel plus gemcitabine. $N$ Engl $J$ Med 2013;369:1691-1703.

24. Von Hoff DD, Ramanathan RK, Borad MJ, et al. Gemcitabine plus nab-paclitaxel is an active regimen in patients with advanced pancreatic cancer: a phase I/II trial. JClin Oncol 2011;29:4548-4554. 25. Socinski MA, Okamoto I, Hon JK, et al. Safety and efficacy analysis by histology of weekly nab-paclitaxel in combination with carboplatin as first-line therapy in patients with advanced nonsmall-cell lung cancer. Ann Oncol 2013;24:2390-2396.

26. Shitara K, Takashima A, Fujitani K, et al. Nab-paclitaxel versus solvent-based paclitaxel in patients with previously treated advanced gastric cancer (ABSOLUTE): an open-label, randomised, non-inferiority, phase 3 trial. Lancet Gastroenterol Hepatol 2017;2:277-287. 